The personalized approach in cancer treatment stimulates the search for new analytical techniques, including spectroscopic methods such as Raman spectroscopy, mass spectrometry MALDI (matrix-assisted laser desorption/ionization) imaging and high-resolution magic angle spinning nuclear magnetic resonance (HR MAS NMR). The purpose of these studies is determination of metabolic profiles of cancer tissues, and their application in diagnostics and therapy of cancers. The review is mainly focused on application of HR MAS NMR technique. Qualitative and quantitative analysis of metabolites by means of this method is described for breast cancer tissues. In the near future HR MAS NMR in vitro studies of metabolic profiles combined with in vivo studies using MRI scanners may be applied as a new diagnostic tool.

Key words: tumor, metabolic profile, nuclear magnetic resonance, MALDI MS imaging, Raman spectroscopy.

\section{Analysis of cancer tissues by means of spectroscopic methods}

\author{
Barbara Pacholczyk ${ }^{1}$, Anna Fabiańska', Renata Kusińska², Piotr Potemski ${ }^{3}$, \\ Radzisław Kordek' ${ }^{2}$, Stefan Jankowski ${ }^{1}$
}

${ }^{1}$ Institute of Organic Chemistry, Faculty of Chemistry, Lodz University of Technology, Poland 2Department of Pathology, Chair of Oncology, Medical University of Lodz, Poland ${ }^{3}$ Department of Chemotherapy, Chair of Oncology, Medical University of Lodz, Poland

\section{Introduction}

For effective treatment of cancers fast selection of therapy is essential. Responses to applied medicines can be different and personalized treatment requires collection of the maximum number of data [1]. One of them is the metabolic profile of the patient reflecting the present state of the organism. For this purpose spectroscopic techniques are applied, such as Raman spectroscopy, mass spectrometry MALDI imaging and high-resolution magic angle spinning nuclear magnetic resonance (HR MAS NMR). These techniques allow determination of the structure of chemical compounds and biomolecules, and are applied in cancer tissue examination. The purpose of these studies is identification of metabolites characteristic for cancer diseases and correlation of changes in metabolic profiles with extent of disease and probable prognosis.

\section{Medical applications of spectroscopic methods}

Raman spectroscopy is based on irradiation of a sample by means of laser and recording of scattered radiation. A significant difference was observed in the Raman spectra of benign and malignant tissues. For cancer tissues bands corresponding to $C-C\left(1150 \mathrm{~cm}^{-1}\right)$ and $C=C\left(1520 \mathrm{~cm}^{-1}\right)$ stretching vibrations of carotenoids and symmetric and asymmetric vibrations of the $\mathrm{C}-\mathrm{H}$ at 2850 $2940 \mathrm{~cm}^{-1}$ for lipids were not observed; however, these bands were present in the spectra of non-cancerous tissues [2]. Diagnostic algorithms [3] were developed on the basis of Raman spectra tissues and tests of imaging were undertaken [4].

Mass spectrometry is based on ionization of the sample and separation of ions according to their mass to charge ratio $(\mathrm{m} / \mathrm{z})$. In analysis of tumor tissues the two-dimensional imaging of cells and tissues by means of MALDI imaging technique was performed. For prostate cancer two-dimensional maps of peptides and proteins containing 350-400 peaks within the range of $\mathrm{m} / \mathrm{z}$ from 2,000 to 20,000 were recorded [5]. Two peptide ions, $m / z$ 4,027 and 4,355, showed significant overexpression in prostate cancer in comparison with benign changes. The peptide ion at $\mathrm{m} / \mathrm{z}$ 4,274 showed expression in benign tissues adjacent to epithelial and stromal cells while for prostate cancer it showed only a little or no expression. The peptide ion at 4,355 was identified as a fragment of mitogen-activated protein kinase - MEKK2 [5].

For breast cancer tissues there were detected six peaks in the range of $\mathrm{m} / \mathrm{z}$ from 8345 to 8640 , more intense in the presence of receptor HER-2. The peak at $m / z 8404$ was identified as cysteine-rich intestinal protein 1 (CRIP1), strongly associated with HER-2 overexpression. Application of MALDI imaging allowed prediction of HER-2 status with a sensitivity of $83 \%$, a specificity of $92 \%$, and an overall accuracy of $89 \%$ [6].

The protein profiles corresponding to early stages of gastrointestinal cancer disease were significantly different from advanced stage tumors. Signals 
identified as $\alpha$-defensin-1, $\alpha$-defensin-2, and calgranulin A and B showed overexpression. Sensitivity and specificity of this method were $93.8 \%$ and $95.5 \%$, respectively [7].

MALDI imaging was applied for 48 tissues from 25 grade III and IV ovarian cancer patients and 23 benign tumors. One of the identified peptides, PA 28 (corresponding to 84 amino acid residues from $11 \mathrm{~S}$ proteasome activator complex), can be used as a cancer marker. PA 28 showed a high level of expression in carcinoma, especially in epithelial cells [8].

Nuclear magnetic resonance (NMR) is a universal technique that allows one to study liquid, solid and gaseous samples placed in the magnetic field. NMR spectroscopy is a nondestructive technique in comparison with mass spectrometry and allows histopathological analysis after measurement.

Today magnetic resonance imaging (MRI) has become a more widely accessible diagnostic technique. The bridge between NMR measurements of liquids and magnetic resonance spectroscopic imaging (MRSI) in vivo by means of MRI scanners is high-resolution magic angle spinning NMR, which was introduced in 1996. The first publication on studies of tissues of lipoma and liposarcoma utilizing this method appeared in 1997 [9]. In 2009 two review articles about tissue spectra registration, identification of metabolites, determination of their concentration and relaxation times (T1 and T2) were published [10, 11].

\section{Basics of HR MAS NMR measurements}

The NMR spectrum is a graph correlating signal intensity and the frequency of radiation absorbed by the atomic nucleus - in the case of tissues, proton atoms. Chemical shifts depend on the structure of the molecule and are expressed in ppm (parts per million). High resolution of the spectrum in HR MAS technique is obtained by spinning the sample around an axis inclined $54.7^{\circ}$ to the direction of the static magnetic field $[12,13]$. Spectra recorded in vivo by means of MRI scanners are of low resolution. Figure 1 illustrates the various methods of examination of the same tissue by NMR technique: $1 \mathrm{H}$ MRSI image and spectrum recorded using a scanner for the selected prostate fragment, which was then excised and re-analyzed. There are clear differences in spectral resolution. The spectrum recorded in vitro provides more information about the kind and concentration of metabolites than the MRSI spectrum.

In HR MAS NMR technique the samples are spun with a spin rate of $4-5 \mathrm{kHz}$ [14]. Too fast spinning may cause mechanical changes and destruction of the sample, while too slow spinning decreases the resolution of spectra [15].

The samples of cancer tissues are frozen at $-80^{\circ} \mathrm{C}$ immediately after excision [16]. It was verified that during prolonged storage at this temperature concentration of metabolites did not change [17]. HR MAS NMR measurements usually are performed at $1-4^{\circ} \mathrm{C}$, but also at room temperature. Tissues taken from rat - fresh excised liver and earlier frozen renal cortex - were examined at $17^{\circ} \mathrm{C}$ and $30^{\circ} \mathrm{C}$ [18]. After excision the liver samples were placed in $\mathrm{D}_{2} \mathrm{O}$ saline on ice and analyzed. No changes were observed in NMR spectra re-recorded over $5 \mathrm{~h}$ after excision. However, significant changes in spectra of the renal cortex recorded after $10 \mathrm{~min}$ and 240 min were observed. Intensities of signals coming from triglycerides and trimethylamine $\mathrm{N}$-oxide were decreased probably due to enzymatic degradation or autolysis [19]. No significant changes in concentrations of $\mathrm{N}$-acetylaspartate, lactate and acetate in rat brain tissues (0-12 min after excision to freezing) were observed. It was concluded that degradation of glucose and glycogen to lactate occurred during the excision of tissue [20]. Reduction of temperature during measurement to $2^{\circ} \mathrm{C}$ reduced changes in concentration of metabolites below 5\% [21].

In the HR MAS NMR method analyzed tissues were placed in special vessels called rotors. The diameter and volume of the rotor were $4 \mathrm{~mm}$ and $50 \mu \mathrm{l}$, respectively. For analysis 8-40 mg of sample is necessary; thus tissues collected by biopsy may also be analyzed. For NMR measurement rotors were filled up with D2O [22] or PBS solution in $\mathrm{D}_{2} \mathrm{O}[23,24]$.

\section{Metabolic profiles of breast cancer determined by HR MAS NMR technique}

Application of HR MAS NMR will be discussed in the example of breast cancer tissues because the most attention was focused on this issue. Cheng et al. were the first to study breast cancer tissues by means of this technique [25]. Reported spectra were dominated by signals from fatty acids. It was found that an increased ratio of lactate and choline concentrations is associated with an increase in tumor grade. It was shown that an increase of $\mathrm{T} 2$ relaxation time of phosphocholine can help to distinguish grade II and grade III of ductal carcinomas. Sitter et al. identified numerous metabolites: amino acids (mainly glycine, alanine, valine, leucine, lysine, isoleucine, tyrosine and histidine), metabolites associated with consumption of energy (lactate, glucose) and choline components (glycerophosphocholine and phosphocholine) [26].

Principal component analysis showed increased levels of lactate, creatine, glycine and phosphocholine from specimens with high numbers of tumor cells. Two groups of samples were studied: tumors smaller than two centimeters (T1) and bigger tumors (T2-T4). The last group of tumors was characterized by higher concentration of choline and glycine. Higher intensity ratios of glycerophosphocholine to phosphocholine and glycerophosphocholine to choline were found for non-involved samples than for tumors, whereas the intensity ratio of phosphocholine to choline was lower for noninvolved specimens [27].

On the basis of microarray studies, basal molecular subtype of breast cancer, an ERBB2-overexpressing group and two or three luminal subtypes A, B and C were distinguished. These subtypes indicated differences in gene expression profile and in prognosis and course of disease [28, 29].

Borgan et al. analyzed the data obtained from HR MAS with microarray studies of gene expression [30]. HR MAS and microarray analysis were performed for 46 samples of breast cancer. The quality of RNA obtained after HR MAS analysis for 11 tissues was insufficient; therefore for microarray analysis adjacent tissue from the same tumors was used. The examined tissues were diagnosed as invasive ductal carcinoma with ER receptor expression which belong to luminal A subgroup. Two strategies were applied to combine the gene expression and metabolic data: multivariate analysis to identify differ- 

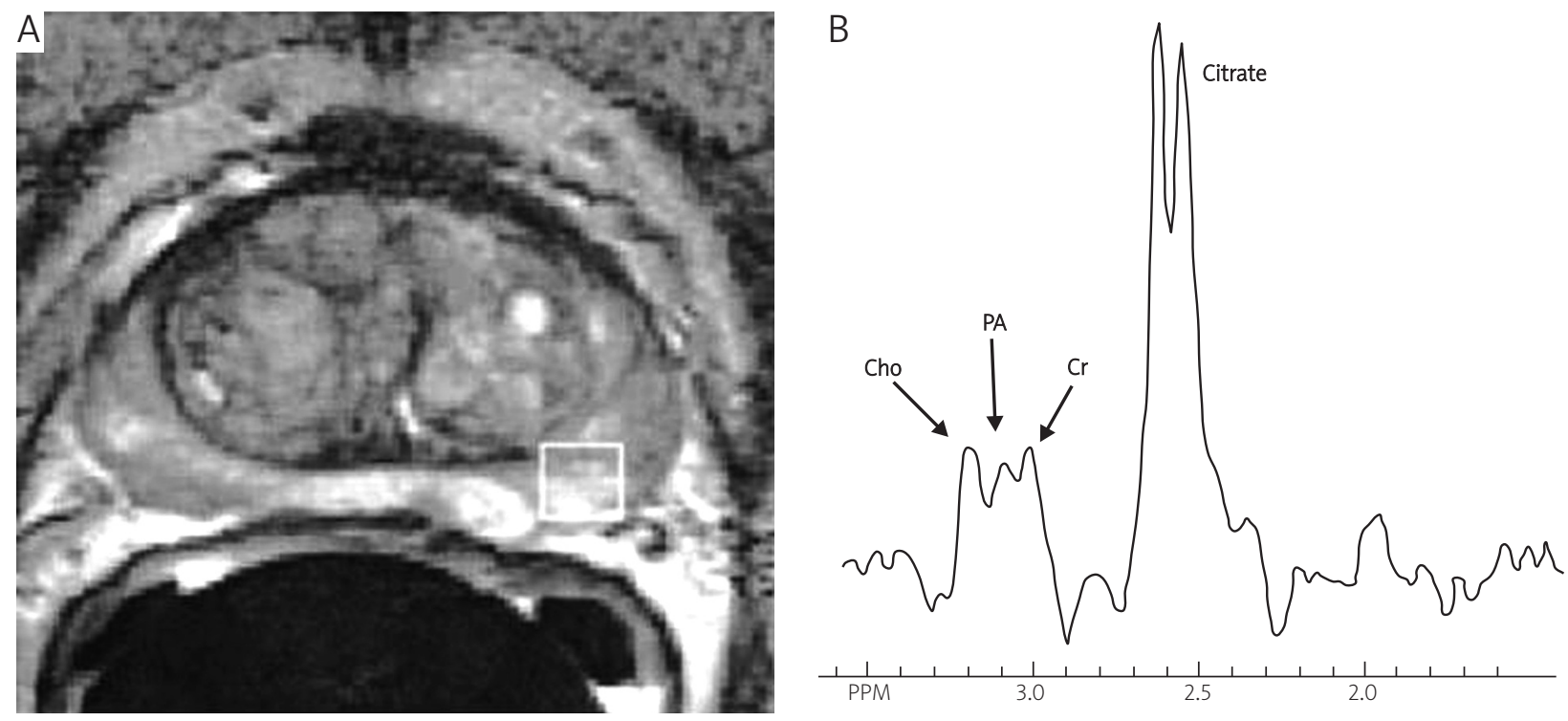

C

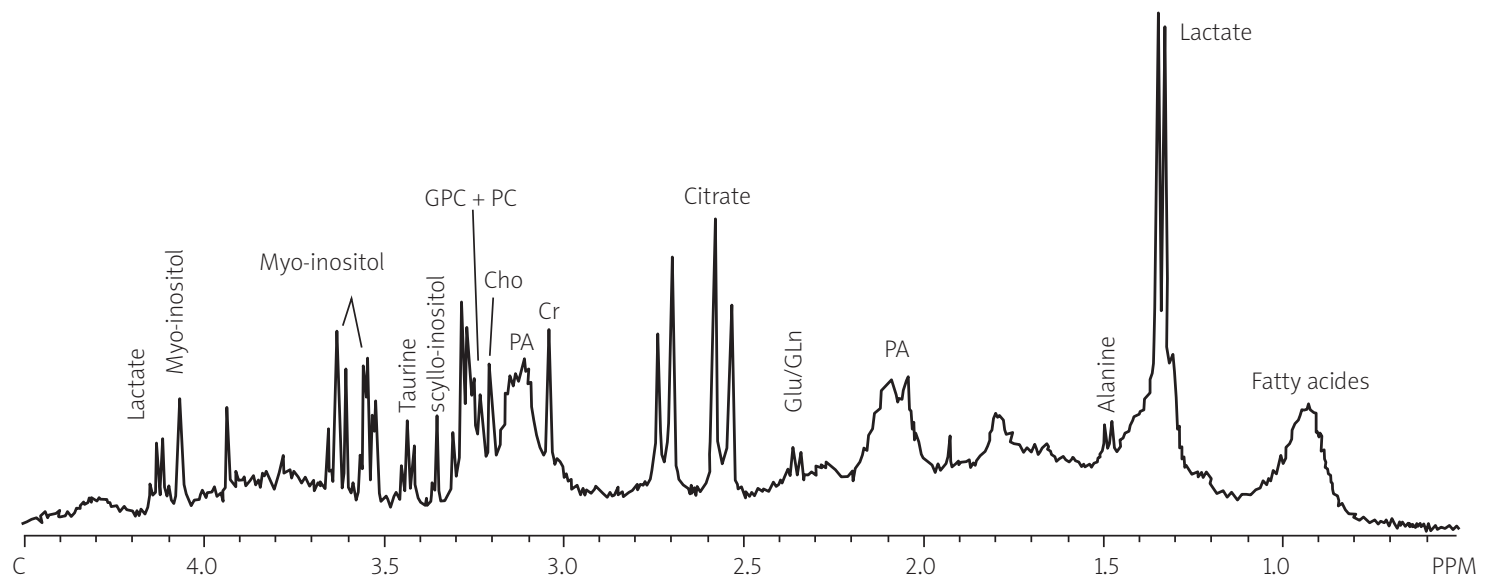

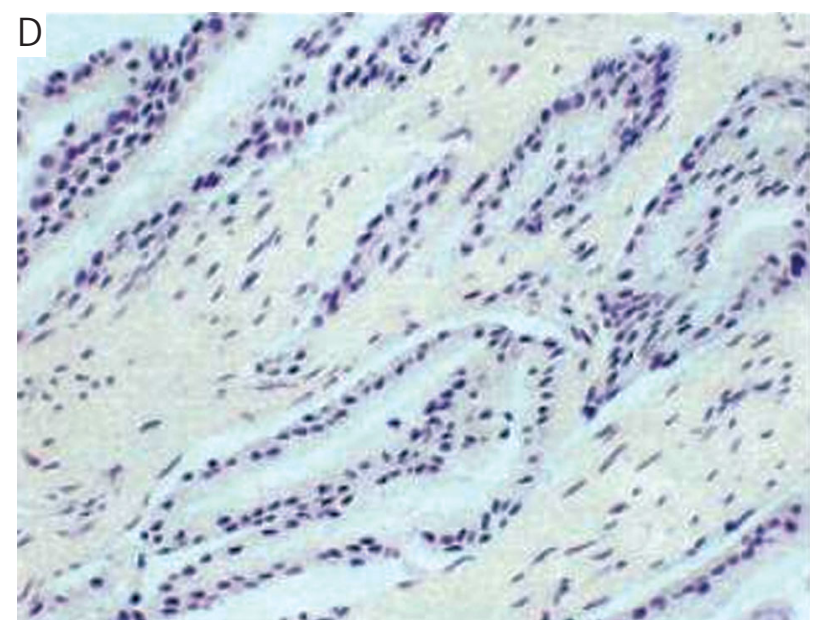

ent groups, and study of the correlation between levels of metabolites and biological processes. On the basis of gene expression 36 tumors were classified as luminal A subtype, one as basal, one as ERBB2, seven normal-like and one unclassified. All samples classified as luminal A were ER positive.
Fig. 1. A - T2-weighted MRI image of prostate; $B$ - MRSI spectrum taken from the voxel shown in $A ; C-1 H$ HR MAS NMR spectrum of excised tissue; D - histological picture of examined specimen, in $60 \%$ contain stromal tissue - H\&E staining

(Cho - choline, Cr-creatine, GPC - glycerophosphocholine,

$P A$ - polyamine, $P C$ - phosphocholine, PPM - parts per million).

[From: Swanson M. G. et al., Magn Res Med 2003; 50: 944-954]
On the basis of HR MAS spectra 31 tissues were classified as luminal A subtype but within this subtype three subgroups were distinguished: $A 1, A 2$ and $A 3$.

In subgroup $A 2$ glucose and alanine concentrations were found to be significantly lower and higher, respectively. 
The intensity of $\alpha$-hydrogen amino acid signals was significantly lower for A1 and higher for A3. Signals of lipid residues were significantly higher and signals of myo-inositol were significantly lower in A1 than in A2 and A3. Moreover, subgroup A2 was enriched for a gene related to the cell cycle and DNA repair. The A2 group's metabolic and transcriptional features indicate a higher Warburg effect and proliferation compared with the other luminal A samples. In the second strategy the correlations between concentrations of myo-inositol, taurine, creatine, glycerophosphocholine, phosphocholine, glycine and choline and all transcripts in microarray were investigated [30].

Concentrations of the amino acids alanine, glycine, leucine, isoleucine, lysine, valine, histidine, tyrosine, glutamate, phenylalanine, taurine and creatine were determined in 80 samples of breast cancer from patients from the Łódź region by the HR MAS NMR method [31]. Moreover, the presence of metabolites such as myo-inositol, scyllo-inositol, choline, phosphocholine, glycerophosphocholine, lactate, acetate and $\beta$-glucose was proven.

A significant increase in the concentrations of creatine, choline, phosphocholine and glycerophosphocholine was found in specimens with increased numbers of cancer cells. Significantly higher concentration of PC was found for patients with higher degree of malignancy. A similar insignificant tendency exists for glycerophosphocholine, phenylalanine and glucose. After the division of samples into three categories (G1, G2 and G3), differences in concentration of phosphocholine disappear and remain only as a strong trend. There was no significant correlation between concentration of phosphocholine and Ki 67 proliferation antigen; howev$\mathrm{er}$, there is a very evident trend. Lower $\mathrm{Cr}$ and Cho concentrations were observed for tumors with metastases in axillary lymph nodes. Reduced amounts of GPC, myo-inositol, glutamate and acetate were found for tumors with estrogen receptor. Only the glutamate level was significantly lower for tumors with the progesterone receptor. Increased level of myoinositol and glutamate accompanies the presence of keratin CK5/6 (tendency for scyllo-inositol and isoleucine). Higher concentrations of $\mathrm{Cr}, \mathrm{GPC}$, acetate and glutamate were found for tumors with protein cyclin E present. A similar tendency was observed for alanine, choline, taurine, glycine, myoinositol, leucine, lysine and reverse for histidine.

There are no significant differences in metabolite concentrations depending on the triple-negative phenotype (tendency towards higher concentrations for phosphocholine and glutamate). Size of tumor, presence of HER2 receptor as well as overall survival do not correlate with any metabolite concentration [31].

\section{Conclusions}

Modern diagnostic methods enable more precise and faster diagnosis of patients with cancer. Application of spectroscopic methods extend the analytical capabilities, in particular nuclear magnetic resonance. It can be expected that the development of nuclear magnetic resonance scanners and higher accessibility of HR MAS NMR measurements would allow us to improve the diagnosis of cancers based on the metabolic profile.
This work was performed within the network "New applications of nuclear magnetic resonance spectroscopy in chemistry, biology, pharmacy and medicine" (68/E-61/BWSN0126/2008). and grants DS/I-18/17/2011 (Institute of Organic Chemistry, LUT), 503/1-034-03/503-1 (Department Tumour Pathology, MUL) and 503/1-034-02/503-1 (Department of (hemotherapy, MUL).

\section{References}

1. Lewandowski R, Roszkowski K, Lewandowska MA. Medycyna personalizowana w onkologii - wizja czy realna koncepcja? Wspolczesna Onkol 2011; 15: 1-6.

2. Abramczyk H, Brożek-Płuska B, Surmacki J, Jabłońska J, Kordek R. The label - free Raman imaging of human breast cancer. J Mol Liq 2011; 164: 123-31.

3. Haka AS, Volynskaya Z, Gardecki JA, et al. Diagnosing breast cancer using Raman spectroscopy: prospective analysis. J Biomed Opt 2009; 14: 054023.

4. Zhang Y, Hong H, Cai W. Imaging with Raman spectroscopy. Curr Pharm Biotech 2010; 11: 654-61.

5. Cazares LH, Troyer D, Mendrinos S, et al. Imaging mass spectrometry of a specific fragment of mitogen-activated protein kinase/extracellular signal-regulated kinase kinase kinase 2 discriminates cancer from uninvolved prostate tissue. Clin Cancer Res 2009; 15: 5541-51.

6. Rauser S, Marquardt C, Balluff B, et al. Classification of HER2 receptor status in breast cancer tissues by MALDI imaging mass spectrometry. J Proteome Res 2010; 9: 1854-63.

7. Kyun KH, Reyzer ML, Choi IJ, et al. Gastric cancer-specific protein profile identified using endoscopic biopsy samples via MALDI mass spectrometry. J Proteome Res 2010; 9: 4123-30.

8. Lemaire R, Menguellet SA, Stauber J, et al. Specific MALDI imaging and profiling for biomarker hunting and validation: fragment of the 115 proteasome activator complex, reg alpha fragment, is a new potential ovary cancer biomarker. J Proteome Res 2007; 6: 4127-34.

9. Millis KK, Maas WE, Cory DG, Singer S. Gradient, high-resolution, magic-angle spinning nuclear magnetic resonance spectroscopy of human adipocyte tissue. Magn Reson Med 1997; 38: 399-403.

10. Sitter B, Bathen TF, Tessem MB, Gribbestad IS. High-resolution magic angle spinning (HR MAS) MR spectroscopy in metabolic characterization of human cancer. Progr Nucl Magn Reson Spectrosc 2009; 54: 239-54.

11. Lindon JC, Beckonert OP, Holmes E, Nicholson JK. High-resolution magic angle spinning NMR spectroscopy: application to biomedical studies. Progr Nucl Magn Reson Spectrosc 2009; 55: 79-100.

12. Andrew ER, Newing RA. The narrowing of nuclear magnetic resonance spectra by molecular rotation in solids. Proc Phys Soc 1958; 72: 959-72.

13. Lowe IJ. Free induction decays of rotating solids. Phys Rev Lett 1959; 2: 285-7.

14. Bathen TF, Jensen LR, Sitter B, Fjosne HE, Halgunset J, Axelson DE, Gribbestad IS, Lundgren S. MR-determined metabolic phenotype of breast cancer in prediction of lymphatic spread, grade, and hormone status. Breast Cancer Res Treat 2007; 104: 181-9.

15. Taylor JL, Wu CL, Cory D, Gonzalez RG, Bielecki A, Cheng LL. Highresolution magic angle spinning proton NMR analysis of human prostate tissue with slow spinning rates. Magn Reson Med 2003; 50: 627-32.

16. Opstad KS, Bell BA, Griffiths JR, Howe FA. Toward accurate quantification of metabolites, lipids, and macromolecules in HRMAS spectra of human brain tumor biopsies using LC model. Magn Reson Med 2008; 60: 1237-42.

17. Jordan KW, He W, Halpern EF, Wu CL, Cheng LL. Evaluation of tissue metabolites with high resolution magic angle spinning MR spectroscopy human prostate samples after three-year storage at $-80^{\circ}$ C. Biomark Insights 2007; 2: 147-54.

18. Tessem MB, Selnæs KM, Sjursen W, Tran $\varnothing \mathrm{G}$, Giske фdegård GF, Bathen TF, Gribbestad IS, Hofsli E. Discrimination of patients with 
microsatellite instability colon cancer using ${ }^{1} \mathrm{~h}$ hr mas $\mathrm{mr}$ spectroscopy and chemometric analysis. J Proteome Res 2010; 9: 3664-70.

19. Waters NJ, Garrod S, Farrant RD, et al. High-resolution magic angle spinning ${ }^{1} \mathrm{H}$ NMR spectroscopy of intact liver and kidney: optimization of sample preparation procedures and biochemical stability of tissue during spectral acquisition. Anal Biochem 2000; 282: 16-23.

20. Tsang TM, Griffin JL, Haselden J, Fish C, Holmes E. Metabolic characterization of distinct neuroanatomical regions in rats by magic angle spinning $1 \mathrm{H}$ nuclear magnetic resonance spectroscopy. Magn Reson Med 2005; 53: 1018-24.

21. Cheng LL, Ma MJ, Becerra L, Ptak T, Tracey I, Lackner A, González RG. Quantitative neuropathology by high resolution magic angle spinning proton magnetic resonance spectroscopy. Proc Natl Acad Sci U S A 1997; 94: 6408-13.

22. Albers MJ, Butler TN, Rahwa I, Bao N, Keshari KR, Swanson MG, Kurhanewicz J. Evaluation of the ERETIC method as an improved quantitative reference for $1 \mathrm{H}$ HR-MAS spectroscopy of prostate tissue. Magn Reson Med 2009; 61: 525-32.

23. Sjøbakk TE, Johansen R, Bathen TF, Sonnewald U, Juul R, Torp SH, Lundgren S, Gribbesta IS. Characterization of brain metastases using high-resolution magic angle spinning MRS. NMR Biomed 2008; 21: $175-85$.

24. De Silva SS, Payne GS, Morgan VA, Ind TEJ, Shepherd JH, Barton DPJ, deSouza NM. Epithelial and stromal metabolite changes in the transition from cervical intraepithelial neoplasia to cervical cancer: an in vivo $1 \mathrm{H}$ magnetic resonance spectroscopic imaging study with ex vivo correlation. Eur Radiol 2009; 19: 2041-8.

25. Cheng LL, Chang I-W, Smith BL, Gonzalez RG. Evaluating human breast ductal carcinomas with high-resolution magic-angle spinning proton magnetic resonance spectroscopy. J Magn Reson 1998; 135: 194-202.

26. Sitter B, Sonnewald U, Spraul M, Fjősne ME, Gribbestad IS. Highresolution magic angle spinning MRS of breast cancer tissue. NMR Biomed 2002; 15: 327-37.

27. Sitter B, Lundgren S, Bathen TF, Halgunset J, Fjosne HE, Gribbestad IS. Comparison of HR MAS MR spectroscopic profiles of breast cancer tissue with clinical parameters. NMR Biomed 2006; 19: 30-40.

28. Perou CM, Sorlie T, Eisen MB, van de Rijn M, Jeffrey SS, Rees CA, Pollack JR, Ross DT. Molecular portraits of human breast tumours. Nature 2000; 406: 747-52.

29. Sorlie T, Perou CM, Tibshirani R, Aas T, Geisler S, Johnsen H, Hastie $T$, Eisen MB. Gene expression patterns of breast carcinomas distinguish tumor subclasses with clinical implications. Proc Natl Acad Sci U S A 2001; 98: 10869-74.

30. Borgan E, Sitter B, Lingiørde OC, Johnsen H, Lundgren S, Bathen TF, Sørlie T, Børresen-Dale AL. Merging transcriptomics and metabolomics - advances in breast cancer profiling. BMC Cancer 2010; 10: 628.

31. Fabiańska A, Pacholczyk B, Kusińska R, Potemski P, Kordek R, Jankowski S, in preparation.

\section{Address for correspondence}

prof. Stefan Jankowski

Institute of Organic Chemistry

Faculty of Chemistry

Lodz University of Technology

Żeromskiego 116

90-924 Łódź

e-mail: stefan.jankowski@p.lodz.pl

Submitted: $\quad 20.12 .2010$

Accepted: $\quad$ 18.01.2012 